OPEN ACCESS

Edited by:

Haike Antelmann,

Freie Universität Berlin, Germany

Reviewed by:

Rute G. Matos,

Universidade Nova de Lisboa,

Portugal

David Herman Bechhofer, Icahn School of Medicine at Mount

Sinai, United States

*Correspondence:

Krishan Gopal Thakur

krishang@imtech.res.in

tPresent address:

Soni Kaundal,

Verna and Marrs McLean Department

of Biochemistry and Molecular

Biology, Baylor College of Medicine,

Houston, TX, United States

Gundeep Kaur,

Department of Epigenetics and Molecular Carcinogenesis, The

University of Texas, MD Anderson

Cancer Center, Houston, TX,

United States

Specialty section:

This article was submitted to Microbial Physiology and Metabolism,

a section of the journal

Frontiers in Microbiology

Received: 14 November 2019

Accepted: 16 January 2020

Published: 14 February 2020

Citation

Kaundal S, Deep A, Kaur G and

Thakur KG (2020) Molecular

and Biochemical Characterization

of YeeF/YezG, a Polymorphic

Toxin-Immunity Protein Pair From

Bacillus subtilis.

Front. Microbiol. 11:95

doi: 10.3389/fmicb.2020.00095

\section{Molecular and Biochemical Characterization of YeeF/YezG, a Polymorphic Toxin-Immunity Protein Pair From Bacillus subtilis}

\author{
Soni Kaundalt , Amar Deep, Gundeep Kaurt and Krishan Gopal Thakur* \\ Structural Biology Laboratory, G. N. Ramachandran Protein Centre, Council of Scientific \& Industrial Research-Institute \\ of Microbial Technology (CSIR-IMTECH), Chandigarh, India
}

Polymorphic toxins are important and widespread elements of bacterial warfare that help in restricting the growth of competitors, aiding kin selection, and shaping the bacterial communities. Although widespread, polymorphic toxin systems (PTS) have been extensively studied in Gram-negative bacteria, there are limited studies describing PTS in Gram-positive bacteria. The present study characterizes YeeF/YezG, a predicted member of a PF04740 family of the polymorphic toxin-immunity system from a Grampositive bacteria Bacillus subtilis. The expression of the C-terminal toxic domain of YeeF (YeeF-CT) causes growth inhibition and gross morphological changes in Escherichia coli. The observed toxic effects are neutralized by the co-expression of yezG, a gene present downstream of yeeF, confirming YeeF-CT/YezG as a toxin/immunity protein pair. Biochemical and in vivo studies reveal that YeeF-CT causes toxicity due to its nonspecific metal-dependent DNase activity. This is different from the previously reported RNase activity from the three B. subtilis toxins belonging to PF04740 family. Isothermal titration calorimetry (ITC) data analysis suggests that YeeF-CT binds YezG with a dissociation constant in the nanomolar range. Analytical ultracentrifugation studies revealed that YeeF-CT forms a homodimer and binds with two molecules of monomeric YezG immunity protein to form a 2:2 stochiometric heterotetrameric complex. Biolayer interferometry and electrophoretic mobility shift assays show that YeeF-CT/YezG/DNA forms a stable ternary complex implicating that YezG is an exosite inhibitor of YeeF-CT. This study extends the molecular targets of the toxins in the PF04740 family and thus, this family of toxins can be broadly classified as nucleases harboring either DNases or RNases activities.

Keywords: contact-dependent antagonism, toxin-immunity system, polymorphic toxin system, bacterial toxin, protein-protein interactions

\section{INTRODUCTION}

Though most of the microbes are uni-cellular, yet they display several complex social traits like communication, swarming motility, regulation of coordinated gene expression, etc., similar to higher multi-cellular organisms. They compete or cooperate with neighboring microbes for limited space and nutrients for survival (Visick and Fuqua, 2005; Keller and Surette, 2006). During 
the course of evolution, microbes have evolved several strategies to communicate, compete or cooperate with each other (Waters and Bassler, 2005; Keller and Surette, 2006; Aoki et al., 2010; Whitney et al., 2017). To restrict the growth of competitors and promote kin selection, microbes secrete several proteinaceous and non-proteinaceous toxic molecules (Zhang et al., 2011, 2012). Polymorphic toxins belong to one of these diverse and widespread family of toxins produced by bacteria that help in restricting the growth of competitors, aiding kin selection, and shaping the bacterial community (Zhang et al., 2011, 2012).

Aravind's group reported comprehensive bioinformatics analyses of polymorphic toxin systems (PTS) (Zhang et al., 2011, 2012) and predicted numerous polymorphic toxin and cognate immunity protein modules in both Gram-positive and Gram-negative bacteria. Polymorphic toxins are multidomain proteins, mainly involved in inter-bacterial conflicts (Zhang et al., 2011, 2012). The product of the immunity gene neutralizes the toxin and protects the toxin-producing cells from auto-inhibition (Zhang et al., 2012). PTS have the following domain characteristics: an N-terminal trafficking domain, a central domain, and a highly variable C-terminal domain. The $\mathrm{N}$-terminal trafficking domain in a toxin is conserved among related toxins belonging to the same family, and is involved in the export of the toxin to the outer membrane (Aoki et al., 2005; Zhang et al., 2011, 2012). The optional central domain varies in length and may contain repeats of several filamentous domains like hemagglutinin, recombinant hotspot, etc. The role of the central domain has not been investigated so far, but probably, this region aids in displaying the toxic module on the cell surface (Aoki et al., 2005; Zhang et al., 2011, 2012). Interestingly, the N-terminal and central regions are conserved in a PTS family, but the C-terminal domain is highly variable and relatively smaller in size harboring toxic activity (Figure 1A). The C-terminal domain displays both sequence and functional diversity with some of the annotated functions including nucleases, pore-forming toxins, nucleic acid modifying enzymes, peptidases, and protein-modifying enzymes such as ADP-ribosyl transferases (Aoki et al., 2005; Zhang et al., 2011, 2012; Jamet and Nassif, 2015). Several polymorphic toxin/immunity families have been predicted in bacteria, but only a few have been characterized experimentally. PTS family includes Contact-Dependent growth Inhibition (CDI) systems (Aoki et al., 2005, 2010), Rearrangement Hot Spot (RHS) toxins (Poole et al., 2011) and multiple adhesion family (maf) toxins (Jamet et al., 2015) that have been studied in Gram-negative bacteria, whereas Pfam PF04740 toxins (Holberger et al., 2012) have been studied in Gram-positive bacteria. Among all of these, $\mathrm{CDI}$ is the most studied phenomenon where $\mathrm{CDI}^{+}$cells inhibit the growth of $\mathrm{CDI}^{-}$cells by direct cell to cell contact. CDI is mediated by $c d i B A I$ gene cluster, where CdiA is the toxin molecule, $\mathrm{CdiB}$ is a predicted outer membrane protein which facilitates the export of CdiA toxin outside the cell surface, and CdiI is the cognate immunity protein (Aoki et al., 2005, 2010; Hayes et al., 2014). Besides these essential components host factor CysK also plays an essential role in mediating CDI in E. coli UPEC536 (Diner et al., 2012; Kaundal et al., 2016). While CDI systems have been reported from Gram-negative bacteria, a few studies refer to the presence of similar systems in Grampositive bacteria as contact-dependent antagonism (Koskiniemi et al., 2013; Jamet et al., 2017, 2018; Whitney et al., 2017). The first report of contact-dependent growth antagonism in Grampositive bacteria was published by Koskiniemi et al. (2013), where WapA polymorphic toxin family of B. subtilis was characterized. The C-terminal domain of WapA protein harbors toxin and is involved in contact-dependent growth antagonism of susceptible strains. Whitney et al. (2017) reported that the LXG toxins (TelB and TelC), secreted by type VII secretion system of Streptococcus intermedius, a Gram-positive bacteria, conferred a competitive advantage against sensitive strains of $S$. intermedius and other strains of Gram-positive species (Streptococcus pyogenes and Enterococcus faecalis). These toxins were then neutralized by the proteins encoded by the genes immediately downstream of telB and telC. Holberger et al. (2012) have distinguished polymorphic toxin modules of Pfam PF04740 family (LXG family) from Gram-positive bacteria. These toxins show the growth inhibitory activity when expressed in E. coli, and coexpression of the cognate immunity protein neutralizes toxicity and prevent autoinhibition. The members of Pfam PF04740 family were characterized and it revealed the RNase activity in YobL-CT, YxiD-CT, and YqcG-CT domains from B. subtilis 168 (Holberger et al., 2012). Although included in the study, the C-terminal toxic domain of YeeF was not functionally characterized (Holberger et al., 2012).

In order to understand the strategies employed by bacteria to outcompete their competitors, we made attempts to characterize one of the uncharacterized toxin-immunity protein modules, a member of Pfam PF04740 polymorphic toxin family, from B. subtilis subsp. spizizenii str. W23. The C-terminal toxic domain from B. subtilis 168 shares $82 \%$ sequence identity with the annotated YeeF-CT from Bacillus subtilis subsp. spizizenii str. W23 characterized in this study. The bioinformatics and the conserved domain database analyses suggests that the $\mathrm{N}$-terminal region of YeeF belongs to a conserved Pfam PF04740, and it harbors a putative nuclease (predicted ribonuclease at UniProt $\mathrm{KB})$ domain in its C-terminal region (Figures 1A,B). We performed detailed biophysical and biochemical investigations on the C-terminal toxic domain of YeeF, henceforth called YeeFCT, a member of the PF04740 toxin system (Figures 1A,B). Here we report that YeeF-CT is a metal ion dependent DNase which is neutralized by $\mathrm{YezG}$, a protein encoded by a gene downstream to yeeF. Hence, YeeF/YezG forms a functional toxin/immunity pair. This study suggests that members of the PF04740 family are nucleases that either have RNase or DNase activity.

\section{MATERIALS AND METHODS}

\section{Cloning of yeeF-CT, yeeF-CT ${ }^{H 581 A}$, and yezG}

DNA region coding for yeeF-CT (residue range Ile527 to Gly669) was cloned in pBADMyc-His A_Modi vector (modified vector where NheI restriction site and N-terminal $6 \times$ His tag is introduced) between NheI and HindIII restriction sites. The yezG gene was cloned between the restriction sites, NdeI/XhoI in 


\section{A Contact-dependent growth inhibition}

\section{CdiB}

Secretion
CdiA

Polymorphic toxin

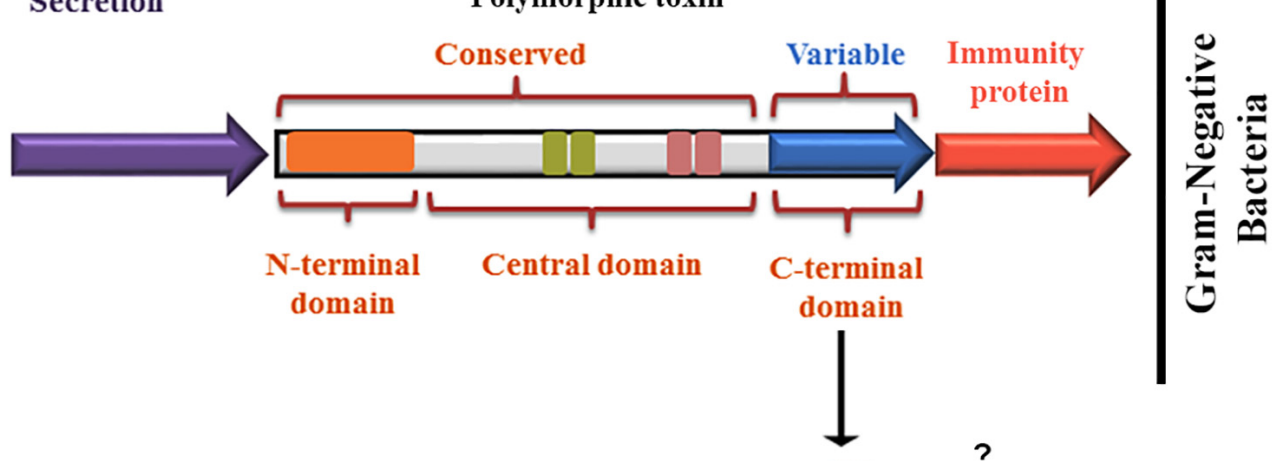

Polymorphic toxic tip

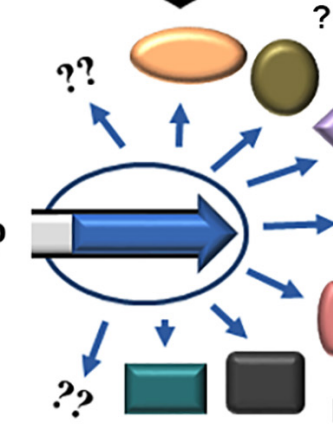

?

\section{CdiI}

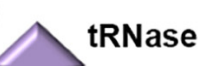

tRnase

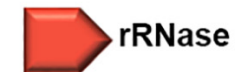

\section{EF-Tu binding}

Pore forming

\section{Contact-dependent antagonism}

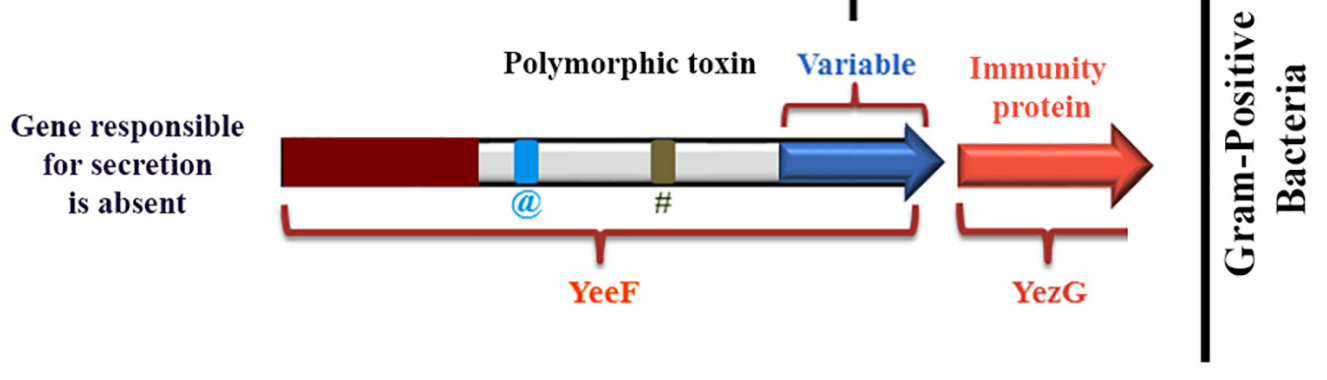

\section{B YeeF domain architecture}

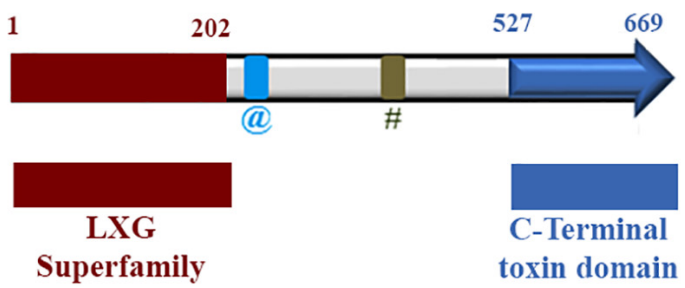

FIGURE 1 | A schematic representation of the polymorphic toxin systems. (A) The schematic representation depicting the organization of polymorphic toxin system involved in contact-dependent growth inhibition in Gram-negative bacteria. An optional dedicated transport system may be encoded in the vicinity of toxin/immunity genes of the toxin. In a defined family, the polymorphic toxin is composed of a conserved N-terminal region having a transporter domain and a variable C-terminal region that codes for the diverse toxin modules. Apart from the transporter domain, the longer central region shows a high variation in the length and contains various combinations of domains/repeats like filamentous hemagglutinin, recombinant hot spot, etc. This central region also presumably helps in toxin display on the cell surface. Operon organization of a polymorphic toxin system involved in contact-dependent antagonism in Gram-positive bacteria. (B) YeeF domain architecture of YeeF toxin. The N-terminal region has a similarity with conserved LXG domain superfamily of Pfam PF04740, which is required for the secretion of toxin by type VII secretion system (Whitney et al., 2017). The C-terminal toxic module is annotated as a putative DNA/RNA non-specific endonuclease. @ Coiled-coil region; \# Low complexity region. Of note that the domain architecture representation is not as per the scale. 
pET28a vector to produce N-terminal $6 \times$ His tagged proteins. The yeeF-CT $T^{H 51 A}$ henceforth called yeeF-CT ${ }^{(M)}$, a point variant was created using PCR based site-directed mutagenesis. The detailed list of constructs used in the present study is provided in the Supplementary Table S1.

\section{Growth Curve and CFU Counting}

Escherichia coli BL21 (DE3) cells expressing YeeF-CT, YeeF-CT ${ }^{(\mathrm{M})}$, YeeF-CT/YezG, and pBADMyc-His A_Modi (negative control) were grown overnight at $37^{\circ} \mathrm{C}$ in $\mathrm{LB}$ media supplemented with appropriate antibiotics. Overnight grown cultures were diluted into a fresh $\mathrm{LB}$ medium to $\mathrm{OD}_{600}$ of 0.07 , then grown to $\mathrm{OD}_{600}$ of 0.4 at $37^{\circ} \mathrm{C}$, and induced with $0.1 \% \mathrm{~L}$-arabinose. The expression of $\mathrm{YezG}$ was induced with $0.3 \mathrm{mM}$ IPTG at $0.2 \mathrm{OD}_{600}$ before the induction of YeeF-CT. The $\mathrm{OD}_{600}$ for each sample was measured and plotted against time. For colony counting, samples were withdrawn at $0,1,2$, and 4-hour post-induction. The ten-fold serial dilutions i.e., $10^{-3}$, $10^{-4}$, and $10^{-5}$ were prepared in LB media and $10 \mu \mathrm{L}$ of the diluted samples were plated on LB agar plates with appropriate antibiotics. The plates were incubated at $37^{\circ} \mathrm{C}$ overnight, and colonies were counted using a colony counter (Heathrow Scientific) and plotted against time. The experiments were done in triplicates. Both the growth curves and CFU data were plotted using Origin software 6.1, and the uncertainties were calculated using standard deviation.

\section{SDS-PAGE Analysis}

Cells were pelleted and lysed using lysis buffer (20 mM HEPES $\mathrm{pH} 7.5,150 \mathrm{mM} \mathrm{NaCl}$, and a cocktail of EDTA-free protease inhibitors) by sonicating the samples for $10 \mathrm{~min}$ and the lysate was fractionated into supernatant and pellet. About $10 \mu \mathrm{g}$ of total protein from supernatant fraction was separated on SDS polyacrylamide gel and detected by staining with Coomassie dye.

\section{Transmission Electron Microscope (TEM)}

Escherichia coli BL21 (DE3) cells expressing YeeF-CT, YeeF$\mathrm{CT}^{(\mathrm{M})}$, YeeF-CT/YezG and pBADMyc-His A_Modi (negative control) were analyzed by TEM. The cells were grown, as mentioned in the above section "Materials and Methods," and were then harvested at $0,30,60$, and 120 min post-induction. Cells were washed twice with $1 \times$ PBS, resuspended in PBS and $30 \mu \mathrm{L}$ of each sample was loaded on the carbon-coated copper grids 300 mesh (Polysciences, Inc.). The grids were then air-dried and loaded in the sample holder and imaged using JEM 2100 transmission electron microscope, operated at $200 \mathrm{keV}$ (JEOL).

\section{Genomic DNA Extraction and in vitro DNase Activity}

The cells expressing YeeF-CT, YeeF-CT ${ }^{(\mathrm{M})}$, YeeF-CT/YezG and pBADMyc-His A_Modi (negative control) were harvested at 12000g. The genomic DNA was isolated using QIAamp DNA mini kit from QIAGEN. The purified DNA samples were resolved on $0.8 \%$ agarose gel. For in vitro DNase activity assays, purified YeeF-CT $(250 \mathrm{nM}-2 \mu \mathrm{M})$ or YeeF-CT ${ }^{(\mathrm{M})}(500 \mathrm{nM}-2 \mu \mathrm{M})$ and were incubated with the plasmid $(\sim 180 \mathrm{ng})$ in the buffer $(20 \mathrm{mM}$
HEPES $\mathrm{pH} 7.5,150 \mathrm{mM} \mathrm{NaCl}$ ) at $37^{\circ} \mathrm{C}$ for $30 \mathrm{~min}$. Then the samples were resolved on $0.8 \%$ agarose gel and visualized in UV transilluminator. To test the metal-dependent DNase activity and identify the metal ion responsible for activating the DNase activity of YeeF-CT, the toxin was chelated with $10 \mathrm{mM}$ EDTA for $2 \mathrm{~h}$ at room temperature and dialyzed against a buffer $20 \mathrm{mM}$ HEPES pH 7.5, $150 \mathrm{mM} \mathrm{NaCl}$ for $12 \mathrm{~h}$. The dialyzed YeeFCT $(2 \mu \mathrm{M})$ was incubated with the mentioned concentration of different metal ions and tested for DNase activity, by being incubated with plasmid DNA for $30 \mathrm{~min}$ at $37^{\circ} \mathrm{C}$. To investigate the protective role of YezG, YeeF-CT $(2 \mu \mathrm{M})$ was pre-incubated with different concentrations of YezG $(0.5-2 \mu \mathrm{M})$ for $45 \mathrm{~min}$ at room temperature and then incubated with DNA. In order to see the optimum time required for DNA cleavage by YeeF$\mathrm{CT}$, the metal-free YeeF-CT supplemented with $10 \mu \mathrm{M} \mathrm{MnCl}_{2}$ was incubated with plasmid DNA ranging from 1 to $60 \mathrm{~min}$ at $37^{\circ} \mathrm{C}$. To investigate the optimum temperature for DNase activity, the metal-free YeeF-CT supplemented with $10 \mu \mathrm{M}$ $\mathrm{MnCl}_{2}$ was incubated with plasmid DNA at 4, 22, 37, and, $55^{\circ} \mathrm{C}$ for $30 \mathrm{~min}$. Similarly, YeeF-CT $(2 \mu \mathrm{M})$ was dialyzed in buffers with different $\mathrm{pH}(4.5-9.0)$ levels and different $\mathrm{NaCl}$ concentrations (0-550 $\mathrm{mM}$ ) for $12 \mathrm{~h}$. The dialyzed protein was incubated with plasmid DNA for $30 \mathrm{~min}$ at $37^{\circ} \mathrm{C}$. The samples in all the above experiments were resolved on $1 \%$ agarose gel and visualized under UV light.

\section{Confocal Microscopy Imaging}

Escherichia coli BL21 (DE3) cells expressing YeeF-CT, YeeF$\mathrm{CT}^{(\mathrm{M})}$, YeeF-CT/YezG, and pBADMyc-His A_Modi (negative control) were used for confocal microscopy. The cells were grown and induced as indicated in the section "Growth Curve and CFU Counting." For confocal microscopy, the samples were washed twice in $1 \times$ PBS and then fixed with $4 \%$ paraformaldehyde for $15 \mathrm{~min}$ at room temperature. Samples were washed twice in $1 \times$ PBS, incubated with DAPI for $30 \mathrm{~min}$ at $37^{\circ} \mathrm{C}$, then washed again with $1 \times$ PBS twice and finally re-suspended in $1 \times \mathrm{PBS}$ and used for confocal microscopy. For confocal microscopy, $10 \mu \mathrm{L}$ of each sample was loaded on the slide coated with $0.7 \%$ agarose, covered with a cover-slip, air-dried and imaged with a confocal microscope (Nikon A1R), using a $100 \times$ oil-immersion objective and 1 Airy unit aperture. Samples were excited using an excitation wavelength of $405 \mathrm{~nm}$.

\section{Protein Purification}

Escherichia coli BL21 (DE3) cells expressing YeeF-CT/YeeF$\mathrm{CT}^{(\mathrm{M})} / \mathrm{YezG}$ were plated on $\mathrm{LB}$ agar plates having specific antibiotics and were incubated at $37^{\circ} \mathrm{C}$ overnight. A single colony from the plate was inoculated in $5 \mathrm{~mL} \mathrm{LB}$ media for primary culture and incubated overnight at a constant shaking of $200 \mathrm{rpm}$ at $37^{\circ} \mathrm{C}$. For secondary culture, $1 \%$ of primary culture was inoculated, and cells were induced by $0.1 \% \mathrm{~L}$ arabinose/0.3 mM IPTG at $0.4-0.5 \mathrm{OD}_{600}$ and further incubated at $37^{\circ} \mathrm{C}$ for $2 \mathrm{~h}$ at a constant shaking of $200 \mathrm{rpm}$. Cells were pelleted by centrifugation at $6000 \mathrm{~g}$ for $15 \mathrm{~min}$ at $4^{\circ} \mathrm{C}$, and the pellets were re-suspended in lysis buffer $(20 \mathrm{mM}$ HEPES $\mathrm{pH} 7.5,150 \mathrm{mM} \mathrm{NaCl}$, and a cocktail of EDTA-free protease inhibitors) followed by sonication. The supernatant was collected 
after centrifugation at $18000 \mathrm{~g}$ for $45 \mathrm{~min}$. All the $6 \times$ His tagged proteins were purified using Ni-NTA based affinity purification method, by following the manufacturer's instructions. The fractions were further loaded and checked on SDS-PAGE before proceeding to the next step. The desired elution fractions were pooled and concentrated using ultrafiltration centrifugal devices (Pall Corporation). Proteins were further purified by gel filtration chromatography using Superdex 200 Increase 10/300 GL column (GE Healthcare). The identities of the protein samples were confirmed by mass spectrometry, and the purities were examined using SDS-PAGE.

\section{Analytical Ultracentrifugation (AUC)}

To determine the oligomeric state of $\mathrm{YeeF}_{-\mathrm{CT}}^{(\mathrm{M})}$ and YezG and their binding stoichiometry, AUC experiments were carried out using Beckman-Coulter XL-A analytical ultracentrifuge equipped/provided with a TiAn50 eight-hole rotor. Sedimentation velocity experiments were conducted using a 2-channel epon centerpiece $(12 \mathrm{~mm})$ and a quartz window. Protein samples were run at three different concentrations $(8,16$, and $32 \mu \mathrm{M}$ ) in buffer $20 \mathrm{mM}$ HEPES, pH 7.5 and $150 \mathrm{mM} \mathrm{NaCl}$ and absorbance scans were recorded at 220, 260, and $280 \mathrm{~nm}$ at every $3 \mathrm{~min}$ interval at $40,000 \mathrm{rpm}$ at $25^{\circ} \mathrm{C}$. To study the binding stoichiometry of YeeF-CT ${ }^{(\mathrm{M})} / \mathrm{YezG}$ complex, purified proteins were mixed (in varying molar ratios as mentioned in section "Result") and incubated at $4^{\circ} \mathrm{C}$ for $1 \mathrm{~h}$ before conducting analytical ultracentrifugation (AUC) experiments. The data were fit by using SEDFIT (Schuck et al., 2002) for continuous distribution c(s). SEDNTERP (Laue et al., 1992) was used to calculate the solvent density $(\rho)$ and viscosity $(\eta)$ from the chemical composition of different components of the buffer.

\section{Isothermal Titration Calorimetry (ITC)}

To determine the $\mathrm{K}_{\mathrm{D}}$, stoichiometry, and thermodynamics of YeeF-CT ${ }^{(\mathrm{M})} /$ YezG binding, ITC experiments were performed using MicroCal VP-ITC (GE Healthcare) at $25^{\circ} \mathrm{C}$. Both the purified proteins were dialyzed using a dialysis membrane of 3500 MWCO against the buffer (20 mM HEPES pH 7.5, $150 \mathrm{mM}$ $\mathrm{NaCl})$. The samples were degassed using MicroCal ThermoVac (GE Healthcare) prior to the experiments. YeeF-CT ${ }^{(\mathrm{M})}(10 \mu \mathrm{M}$; sample cell) was titrated with YezG (300 $\mu \mathrm{M}$; syringe) with a constant stirring speed of $307 \mathrm{rpm}$. The reference power and injection volume were kept $5 \mu \mathrm{cal} / \mathrm{sec}$ and $6 \mu \mathrm{L}$, respectively. Since $\mathrm{YeeF}-\mathrm{CT}{ }^{(\mathrm{M})}$ is a dimer as per AUC results, two binding site models were used to analyze protein-protein interactions. The binding isotherms were fit to determine apparent molar reaction enthalpy $(\Delta H)$, apparent entropy $(\Delta S)$, dissociation constant $\left(K_{\mathrm{D}}\right)$ and stoichiometry of binding $(N)$. To calculate ligand heat of dilution, experiments were performed under the same experimental conditions, except the protein in the sample cell was replaced with the buffer. In all the calculations, the ligand heat of dilution was subtracted from the data. The data was analyzed using Origin 6.0 software suite.

\section{Bio-Layer Interferometry (BLI)}

To study the interactions of YeeF-CT ${ }^{(\mathrm{M})}$ with DNA, ForteBio Octet RED 96 (Forte Bio, Fremont, CA, United States) and streptavidin-coated (SA) sensors (Forte Bio, Fremont, CA, United States) were used. The experiment was conducted at $25^{\circ} \mathrm{C}$ in $20 \mathrm{mM}$ HEPES $\mathrm{pH} 7.5,150 \mathrm{mM} \mathrm{NaCl}$ as assay buffer, 30 mer $5^{\prime}$ biotinylated DNA was synthesized (5'-AGC ACAATTTAACACTTTTGTCAAGCGGCC-3') and annealed with complementary DNA in the annealing buffer $(20 \mathrm{mM}$ Tris $\mathrm{pH}$ 8.0, $50 \mathrm{mM} \mathrm{NaCl}$ and $5 \mathrm{mM}$ EDTA). Before use, the SA sensor tips were hydrated in assay buffer for $15 \mathrm{~min}$. The wells in 96-microwell plate were filled with $200 \mu \mathrm{L}$ of either buffer or sample in each case and agitated at $1000 \mathrm{rpm}$. $0.6 \mathrm{~nm}$ biotinylated double-stranded DNA was immobilized on the hydrated sensor tips (working and reference). Another reference sensor without biotinylated double stranded DNA subjected to the same procedure for double referencing to subtract non-specific protein binding with streptavidin-coated sensors. Following immobilization with biotinylated DNA, binding interaction with different concentrations $(0.25-2 \mu \mathrm{M})$ of YeeF-CT ${ }^{(\mathrm{M})}$ was carried out, which included baseline $(120 \mathrm{~s})$, association (300 s), dissociation (600 s), regeneration with $10 \mathrm{mM}$ $\mathrm{NaOH}$ and (30 s) baseline (120 s). To measure the binding kinetics of YeeF-CT ${ }^{(\mathrm{M})}$ with DNA, the sensor tip immobilized with DNA was first moved to the well, containing assay buffer with YeeF-CT ${ }^{(\mathrm{M})}$, to study the association and then moved to well, having only assay buffer to study dissociation. $\mathrm{K}_{\mathrm{D}}$ was calculated using the curve fit (1:1) model using ForteBio Data Analysis 9.0 software.

\section{Electrophoretic Mobility Shift Assay (EMSA)}

For in vitro DNA binding analysis, electrophoretic mobility shift assays were performed. Varying concentrations of YeeF$\mathrm{CT}^{(\mathrm{M})}$ and YeeF-CT ${ }^{(\mathrm{M})} / \mathrm{YezG}(12.5-50 \mu \mathrm{M})$ were mixed with 50 and 80 ng of dsDNA (PCR amplified product) in buffer (20 mM HEPES pH 7.5, $150 \mathrm{mM} \mathrm{NaCl,} 5 \mathrm{mM}$ EDTA) followed by incubation at room temperature for $30 \mathrm{~min}$. The samples were resolved on 1\% agarose gel, stained with ethidium bromide and visualized using gel documentation system (Syngene, United States).

\section{RESULTS}

\section{The Expression of YeeF-CT Is Toxic for Bacterial Growth and YezG Co-expression Can Neutralize the Toxic Effect}

To functionally characterize YeeF-CT toxin, we transformed pBADMyc-His A-Modi-yeeF-CT plasmid into E. coli BL21(DE3) competent cells. The extracellular export of polymorphic toxins is mediated by two component secretion systems in Gram-negative bacteria and by type VII secretion system in Gram-positive bacteria. The N-terminal domain in CDI and contact dependent antagonism toxins plays a crucial role in toxin secretion (Aoki et al., 2005; Whitney et al., 2017). However, to test toxicity of YeeF-CT in the heterologous host, E. coli, the N-terminal domain was not included in the construct. The cognate immunity 
proteins on the other hand are cytosolic proteins as their primary role is to protect host cells from autoinhibition (Aoki et al., 2005; Whitney et al., 2017). So, we used native full length YezG for co-expression and neutralization studies. The expression of YeeF-CT was induced with $0.1 \% \mathrm{~L}$-arabinose. The growth curve results show that induction of the YeeF-CT inhibits the growth of $E$. coli when compared to both uninduced cells and cells carrying empty vector (negative control) (Figure 2A). The protein expression was confirmed by SDS-PAGE analysis (Figure 2A, inset). To investigate the protective role of $\mathrm{YezG}$, we co-transformed pET28a-yezG and pBADMyc-His A_Modi-yeeF$C T$ for the co-expression of both YezG and YeeF-CT, respectively. Cells co-expressing YeeF-CT and YezG had a similar growth curve profile as control cells (Figure 2A), indicating that coexpression of YezG protects against YeeF-CT mediated toxicity. Histidine is a catalytically important residue in several nucleases (Ho et al., 2000). Multiple sequence alignment of YeeF-CT with homologs in other bacteria suggest that His581 is highly conserved (Supplementary Figure S1). So, we mutated His581 residue to Alanine to test its potential role in the activity and constructed YeeF-CT ${ }^{(\mathrm{M})}$ point variant. The expression of YeeF$\mathrm{CT}^{(\mathrm{M})}$ was confirmed by SDS-PAGE analysis (Figure 2A, inset). The growth curve assays show that the expression of $\mathrm{YeeF}_{-\mathrm{CT}}{ }^{(\mathrm{M})}$ had no visible effect on the E. coli growth (Figure 2A).

Though the optical density based growth curve analysis suggests that expression of YeeF-CT causes growth arrest, we further wanted to determine the effect of YeeF-CT expression on cell viability. We performed colony forming unit (CFU) assay to study cell viability. The data suggests that the expression of YeeF-CT resulted in CFU reduction after 1-hour post-induction (Figure 2B). The CFU counts in the negative control cells, cells expressing YeeF-CT ${ }^{(\mathrm{M})}$, and cells co-expressing YeeF-CT and YezG showed no reduction in cell viability (Figure 2B). The reduction in CFU counts suggested that YeeF-CT expression is bactericidal (Figure 2B). We did not notice any observable changes in the growth curves in the initial hours postinduction, but there was a reduction in the CFU counts in the cells expressing YeeF-CT. OD based measurements do not differentiate live $v s$ dead cells hence, CFU based experiments were crucial to examine the effect of toxin expression. Further, we were interested in investigating the effect of YeeF-CT expression on cell morphology. Therefore, we used transmission electron microscopy (TEM) to visualize the effect of YeeFCT expression on the cellular morphology. The TEM imaging of cells expressing YeeF-CT, YeeF-CT ${ }^{(\mathrm{M})}$, YeeF-CT/YezG and cells harboring negative control was performed at four-time points i.e., 0, 30, 60, and $120 \mathrm{~min}$ post-induction (Figure 2C). At initial time points, all the cells showed normal rod-shaped morphology with intact cell membrane. The toxic effect of YeeFCT resulted in gross morphological defects as indicated by irregular cell surfaces, membrane disruption and, cell elongation (Figure 2C). Interestingly, the cells expressing $\mathrm{YeeF}_{-} \mathrm{CT}^{(\mathrm{M})}$, YeeF-CT/YezG and carrying vector only show intact cells with a typical rod-shaped morphology at all the time points tested in the current study. With the increase in time, there are pronounced differences in the morphological features of the cells expressing YeeF-CT as compared to the cells expressing
YeeF-CT ${ }^{(\mathrm{M})}$ and YeeF-CT/YezG toxin/immunity pair. Together these results confirmed that YeeF-CT expression was toxic to the cells and induced observable morphological changes. Also, YeeF-CT and YezG constitute a functional toxin/immunity pair, thereby demonstrating the potential role of YezG in protecting inhibitor cells from autoinhibition.

\section{YeeF-CT Is a Non-specific DNase and Cleaves DNA in Both in vitro and in vivo Conditions}

To understand the molecular basis of toxicity caused by YeeFCT in the target cells, we attempted to identify the molecular target(s) of YeeF-CT toxin. YeeF-CT is a predicted non-specific DNA/RNA endonuclease, so we investigated DNase and RNase activities. To identify the molecular target, we isolated the genomic DNA (gDNA) and total RNA from the cells expressing YeeF-CT. We could clearly observe DNA smearing in the cells over-expressing YeeF-CT while the gDNA profiles of samples before induction and negative control were similar (Figure 3A). This data suggests that YeeF-CT cleaves DNA (Figure 3A). There was no observable change in the total RNA extracted UREAPAGE profiles of all the samples suggesting YeeF-CT does not cleave RNA (Supplementary Figure S2). The gDNA profile of the samples expressing both YeeF-CT and YezG shows gDNA profile similar to the negative control suggesting the protective role of YezG in YeeF-CT mediated toxicity (Figure 3A). To investigate whether the DNA cleavage in YeeF-CT induced toxicity is a consequence of the direct activity of YeeF-CT, we performed in vitro DNA cleavage assays using supercoiled plasmid DNA as substrates. YeeF-CT was able to degrade the supercoiled plasmid in a concentration-dependent manner (Figure 3B). To investigate the protective role of $\mathrm{YezG}$ in vitro, we first incubated YeeF-CT with increasing concentrations of YezG at room temperature for $45 \mathrm{~min}$ and then mixed this preformed complex with DNA. The results show that YezG was able to protect DNA degradation in a concentration-dependent manner (Figure 3C) further suggesting the role of YezG immunity protein as a direct inhibitor of YeeF-CT's DNase activity. The purified YeeF-CT ${ }^{(\mathrm{M})}$ did not show the digestion of plasmid DNA (Figure 3D).

We performed confocal microscopy experiments to further probe the in vivo DNase activity of YeeF-CT. Cells were stained using DAPI staining. Cells transformed with pBADMyc-His A_Modi (negative control), pBADMyc-His A_Modi-yeeF-CT (active toxin), pBADMyc-His A_Modi-yeeF-CT ${ }^{(\mathrm{M})}$ (inactive toxin), pBADMyc-His A_Modi-yeeF-CT/pET28a-yezG (coexpression of toxin and immunity protein) were induced by adding appropriate inducer. Negative control cells, cells expressing YeeF-CT ${ }^{(\mathrm{M})}$, and YeeF-CT/YezG exhibited uniform DNA staining indicating intact DNA before induction and 4-hour post-induction, while fluorescence signal was highly reduced in cells expressing active toxin suggesting extensive degradation of DNA (Figure 3E). The quantitative analysis of fluorescence signals also suggests a reduction in the fluorescence signals obtained from the cells expressing YeeF-CT (Figure 3F). These results indicate that the expression of YeeF-CT causes 


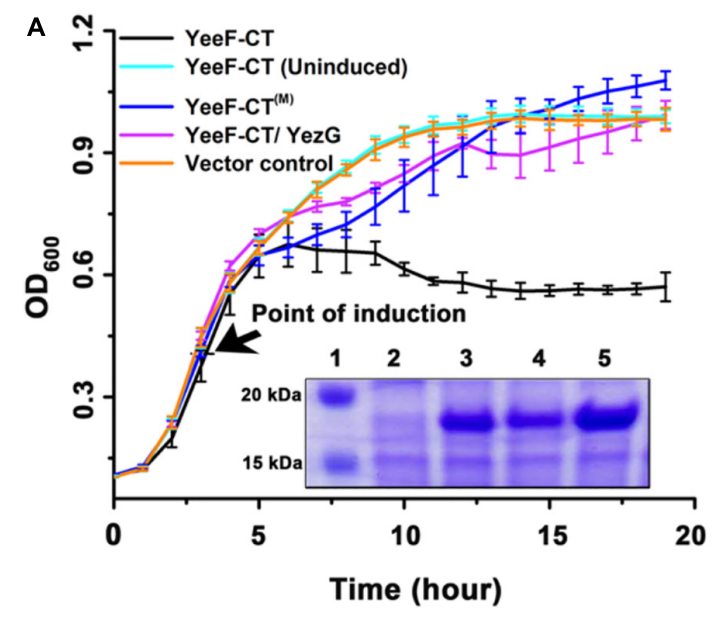

C
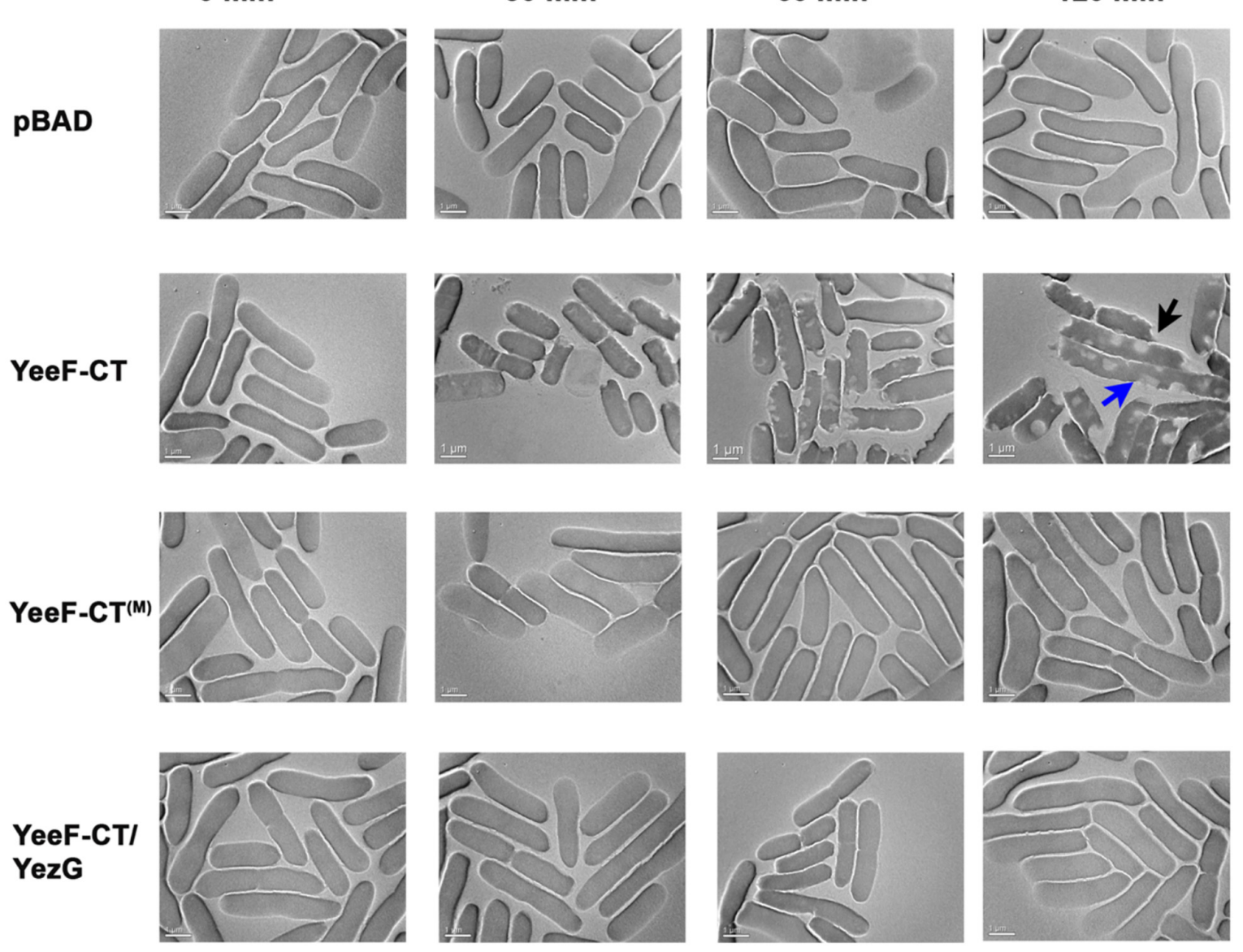

FIGURE 2 | Effect of YeeF-CT expression on bacterial growth and morphology. (A) The growth curve assay was performed in cells expressing YeeF-CT, YeeF-CT(M) and YeeF-CT/YezG and negative control. Cells expressing YeeF-CT shows reduction in $\mathrm{OD}_{600}$ whereas YeeF-CT ${ }^{(\mathrm{M})}$, YeeF-CT/YezG, and negative control expression did not affect cell growth. The inset shows SDS-PAGE analysis showing the expression of proteins; (1) Protein ladder; (2) Negative control; (3) YeeF-CT; (4) YeeF-CT ${ }^{(M)}$ and; (5) YeeF-CT/YezG. Since the molecular weight of YeeF-CT and YezG are apparently similar i.e., 18.9 and 19.2 kDa, respectively, so they appeared as a single band. (B) The CFU counting analysis also correlates well with the growth curve analysis. (C) TEM imaging of cells harboring plasmid only (upper panel), expressing YeeF-CT (second panel), YeeF-CT ${ }^{(M)}$ (third panel) and YeeF-CT/YezG (lower panel). While cells imaged at 0 min show smooth surface in all the samples, 30 min post-induction, cells expressing YeeF-CT appears to have some defects on the surface, which becomes quite evident at the 60 and 120 min post-induction. The blue arrow shows cell elongation and black arrow shows a defect in the cell surface. The cells expressing YeeF-CT ${ }^{(M)}$ and YeeF-CT/YezG appeared to have a smooth surface and intact cell wall at all the time points. 


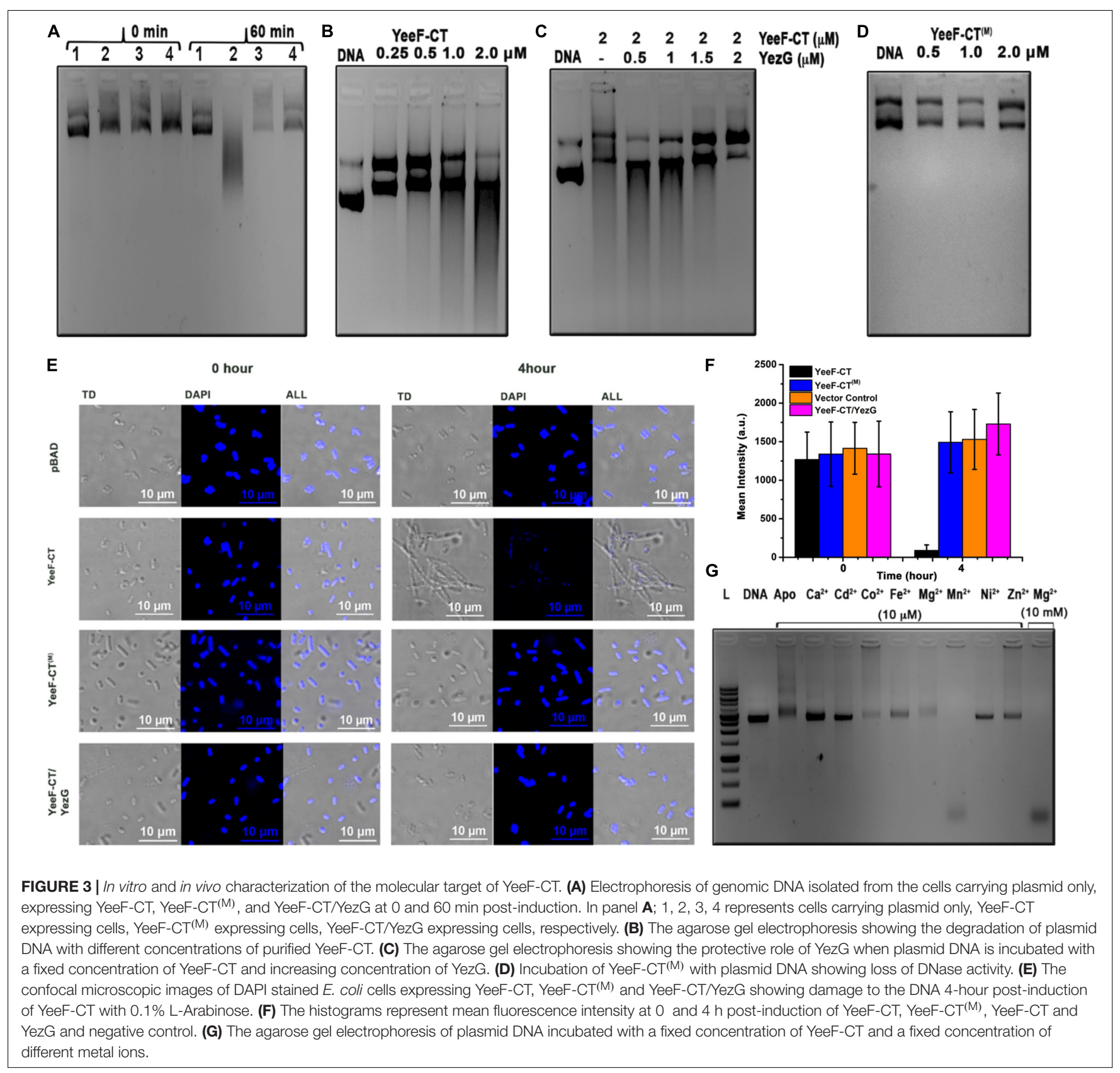

toxicity by degrading gDNA of the cells, which could be rescued by the expression of YezG. Also, these studies further confirm the role of highly conserved His581 residue in the DNase activity of YeeF-CT.

\section{YeeF-CT Is a Metal-Dependent DNase}

To investigate whether YeeF-CT is a metal-dependent DNase, we prepared apo-YeeF-CT by chelating metal ions from purified protein with $10 \mathrm{mM}$ EDTA. The result suggests that apo-YeeFCT does not show any DNase activity (Figure 3G) indicating that YeeF-CT is a metal-dependent DNase. However, there was upward smearing probably due to the formation of YeeF-CT: DNA complex formation. To identify the metal ion(s) responsible for DNase activity, we incubated apo-YeeF-CT with $10 \mu \mathrm{M}$ concentration of different alkaline, earth and transition divalent metal ions. The agarose gel electrophoresis shows that depending on the metal ion supplemented, a varying degree of DNA cleavage was observed. YeeF-CT displayed robust DNA cleavage with $\mathrm{Mn}^{2+}$ at $10 \mu \mathrm{M}$ physiological concentration (Anjem et al., 2009; Kaur et al., 2014). The intracellular concentration of $\mathrm{Mg}^{2+}$ is in $\mathrm{mM}$ range (Cayley et al., 1991), so we also tested the activity in the presence of $10 \mathrm{mM} \mathrm{Mg}^{2+}$, the catalytic activity was equivalent to that observed for $\mathrm{Mn}^{2+}$. For further biochemical characterization of YeeF-CT, we investigated its DNase activity at a different time, a different temperature, a different $\mathrm{pH}$ level, and different salt concentrations using purified plasmid as a substrate. Our data 
suggest that YeeF-CT shows time-dependent cleavage of DNA substrate (Supplementary Figure S3A). The enzyme showed activity at a wide temperature range $\left(4\right.$ to $55^{\circ} \mathrm{C}$ tested in this study), with the highest activity at $37^{\circ} \mathrm{C}$. At $4^{\circ} \mathrm{C}$ we could observe a population of the relaxed nicked DNA suggesting a nickase activity (Supplementary Figure S3B). The enzyme is active in the basic $\mathrm{pH}$ range and up to $250 \mathrm{mM} \mathrm{NaCl}$ concentration (Supplementary Figures S3C,D).

\section{YeeF-CT Exists as a Homodimer in Solution and Interacts With Monomeric YezG With Nanomolar Range Dissociation Constant}

The oligomeric state plays an important role in the function of a protein. We used analytical ultracentrifugation (AUC) to examine the oligomeric states and binding stoichiometry of the YeeF-CT and YezG. AUC experiments were performed at three different concentrations of both the purified proteins at $25^{\circ} \mathrm{C}$. Sedimentation velocity analysis of the purified YeeF-CT and YezG revealed a major population of 2.6 and $2.1 \mathrm{~S}$, respectively. Suggesting YeeF-CT is a homodimer across the range of concentrations tested (Figure 4A) and YezG sediments as a monomer (Figure 4B). Additionally, concentration-dependent higher-order oligomers formation was observed as a minor population $(\sim 3-5 \%)$ in both the proteins. To determine the binding stoichiometry of the complex, the concentration of YeeF-CT was fixed and titrated with increasing concentrations of YezG. A single peak corresponding to $3.4 \mathrm{~S}$ was observed when $7.5 \mu \mathrm{M}$ of YeeF-CT (dimer concentration) was mixed with $15 \mu \mathrm{M}$ of YezG (monomer concentration) indicating that a dimeric toxin molecule binds with two monomeric immunity protein molecules. (Figure 4C). Detailed parameters derived from the AUC experiments are listed in Supplementary Table S2. To further characterize YeeF-CT and YezG interactions, we used isothermal titration calorimetry (ITC). Since YeeF-CT is a homodimer and YezG exists as a monomer, we used 2-site binding to fit ITC data. The binding isotherm showed a clear biphasic binding profile suggesting cooperativity in the proteinprotein interactions. The ITC data suggest the two binding sites in YeeF-CT have different affinities $\mathrm{K}_{\mathrm{D} 1}=29.4 \pm 4.7$ and $\mathrm{K}_{\mathrm{D} 2}=91.7 \pm 7.3 \mathrm{nM}$ for YezG (Figure 4D). Since YeeF-CT is a homodimer, hence there are two potential YezG binding sites and the binding of the first YezG molecule allosterically affects the binding of the other molecule. All the thermodynamic parameters are summarized in Supplementary Table S3.

\section{YeeF-CT/YezG/DNA Forms a Stable Ternary Complex: YezG Is an Exosite Inhibitor of YeeF-CT}

Using YeeF-CT ${ }^{(\mathrm{M})}$ active site mutant of YeeF-CT, we performed electrophoretic mobility shift assays to investigate its DNA binding property. We observed retardation in the mobility of DNA caused by binding of $\mathrm{YeeF}-\mathrm{CT}^{(\mathrm{M})}$ in a concentrationdependent manner (Figure 5A). With the increase in the concentration of YeeF-CT ${ }^{(\mathrm{M})}$, the DNA/protein complex was unable to migrate from the wells. We further validated the DNA binding property of YeeF-CT using BioLayer Interferometry (BLI) experiments. The DNA was immobilized on the SA sensor chip. Shifts in the sensorgrams were observed during the association phase with $\mathrm{YeeF}_{-\mathrm{CT}}{ }^{(\mathrm{M})}$ (Figure 5B). We also investigated the DNA binding property of $\mathrm{YeeF}^{-C T}{ }^{(\mathrm{M})}$ in the presence of YezG to see whether the binding of YezG inhibits substrate binding. Interestingly, the preformed complex of YeeF-CT/YezG retained the ability to bind DNA, suggesting DNA binding site on YeeF-CT did not mask the binding of YezG (Figure 5C). Upon adding the different concentrations of preformed YeeF-CT/YezG complex, we could observe a shift in the sensorgrams suggesting complex retains the ability to bind DNA (Figure 5D). YeeF-CT is a non-specific DNase so the stoichiometry of YeeF-CT ${ }^{(\mathrm{M})}$ : DNA fragment used in the study is difficult to interpret as per the performed experiments. The BLI results are in good agreement with EMSA studies. Together, these results indicate that YeeF-CT retains DNA binding property in both free and YezG bound states. In other words, YezG and/or DNA binds on non-overlapping sites of YeeF-CT. Thus, YezG acts as an exosite inhibitor of YeeF-CT activity.

\section{DISCUSSION}

Microbes are always under constant threat from neighboring microbial competitors for limited resources and space (Hibbing et al., 2010). A recent report has demonstrated that members of the LXG family of polymorphic toxins are involved in the contact-dependent growth antagonism which may play an important role in interbacterial competition (Whitney et al., 2017). In the present study, we performed a detailed investigation on YeeF-CT/YezG, a member of the LXG family of polymorphic toxins, from B. subtilis subsp. spizizenii str. W23. Our results suggest that the heterologous expression of the YeeF-CT inhibits cell growth which can be blocked by the expression of YezG, a cognate immunity protein. The cytotoxicity of YeeF-CT can be attributed to its non-specific DNase activity targeting chromosomal DNA, probably beyond repair by DNA repair machinery. Although polymorphic toxins are predicted to target a diverse range of molecular targets (Zhang et al., 2011, 2012; Holberger et al., 2012), most of the experimentally characterized polymorphic toxins have nuclease activity targeting either DNA or RNA (Aoki et al., 2010; Diner et al., 2012; Morse et al., 2012; Jamet and Nassif, 2015; Johnson et al., 2016b; Ogier et al., 2016). The polymorphic toxins belong to different super-families like CdiA-CT ${ }^{\mathrm{ECL}}$ toxin from Enterobacter cloacae ATCC 13047 and CdiA-CT ${ }^{\text {EC536 }}$ from uropathogenic E. coli 536 are members of Ntox21 and Ntox28 super families, respectively (Johnson et al., 2016a,b). These toxins differ in substrate specificity, and most of them are $\mathrm{Mg}^{2+}$ and/or $\mathrm{Zn}^{2+}$ dependent nucleases (Morse et al., 2012; Johnson et al., 2016a,b) with an exception of CdiA-CT ${ }^{\text {Ykris }}$ from Y. kristensenii ATCC 33638 which is a metal independent nuclease (Batot et al., 2017). The polymorphic toxins utilize only one metal ion for their nuclease activity (Morse et al., 2012; Johnson et al., 2016a,b) with exception of CdiA-CT ${ }^{\text {EC869, }}$ which utilizes $\mathrm{Mg}^{2+}$ for nickase activity and $\mathrm{Zn}^{2+}$ for DNase activity (Morse et al., 2012). In the previous study, members of 

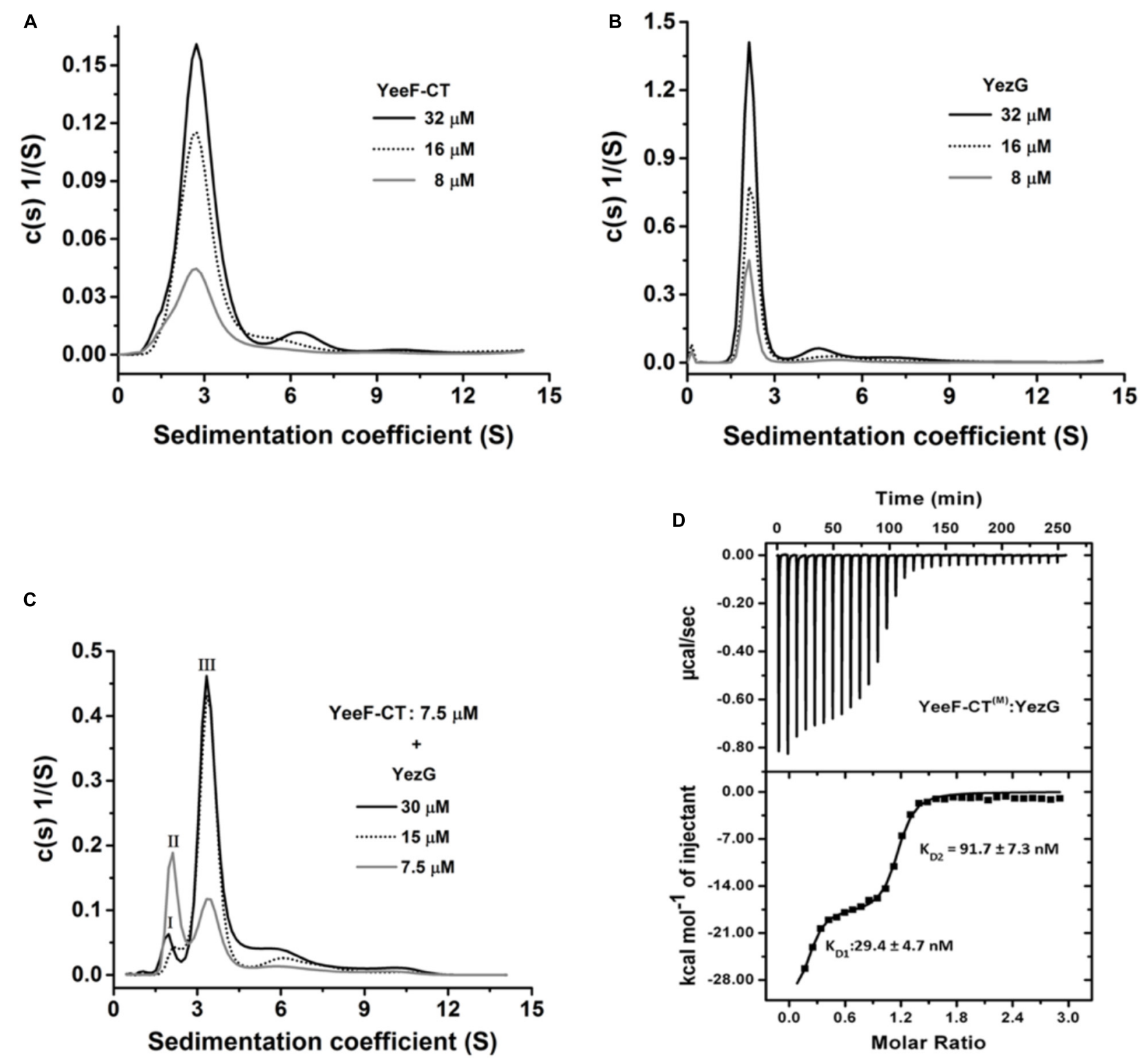

FIGURE 4 | Oligomeric state and binding kinetics of YeeF-CT, YezG, and their interactions. Sedimentation coefficient distribution (s) of YeeF-CT, YezG, and YeeF$\mathrm{CT} /$ YezG are shown in panels A-C, respectively. (A,B) YeeF-CT and YezG exist predominantly as a homodimer and monomer in solution, respectively. (C) YeeF-CT/YezG associate to form a heterotetramer in solution. Peak I and II correspond to excess YezG and YeeF-CT, respectively. Peak III corresponds to YeeF-CT/YezG complex. In all the protein samples, a minor concentration-dependent higher order oligomer population is also observed. (D) A representative isotherm profile of YeeF-CT and YezG interactions. The upper panel represents the calorimetric titration and the lower panel represents derived binding isotherm plotted versus the molar ratio of the YezG.

the PF04740 family revealed RNase activity in YobL-CT, YxiDCT and YqcG-CT domains from B. subtilis 168 (Holberger et al., 2012). However, YeeF-CT from B. subtilis 168 was not functionally characterized. In this study, we show that YeeF-CT is a metal-dependent DNase and requires $\mathrm{Mg}^{2+}$ or $\mathrm{Mn}^{2+}$ ion(s) for its activity. Further biochemical characterization of YeeFCT suggests that this enzyme is active in diverse conditions like elevated temperature, alkaline $\mathrm{pH}$ and low salt conditions. So, the data presented here add DNA to the list of the molecular targets of PF04740 family toxins and hence this family of toxins can be broadly classified as nucleases having either RNase or DNase activities.

In this study, we demonstrate that DNA/YeeF-CT/YezG can form a ternary complex indicating substrate binding site and the immunity protein binding sites are spatially quite distinct. This kind of immunity proteins are termed as "exosite" inhibitors of enzymes (Kleanthous and Walker, 2001). Exosite is the secondary binding site distant from the active site present in many enzymes (Kleanthous and Walker, 2001). This mode of inhibition is also present in $\mathrm{CdiA}-\mathrm{CT}_{o 11}{ }^{\mathrm{EC} 869} / \mathrm{CdiI}$ toxin immunity complex 


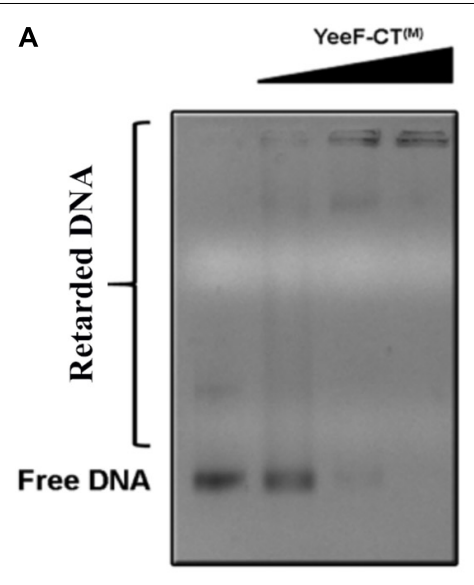

C

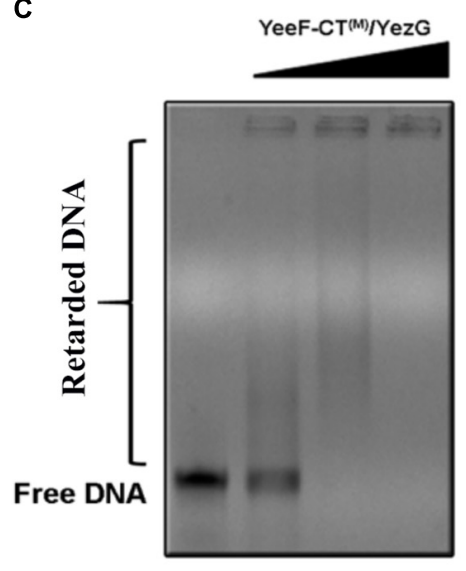

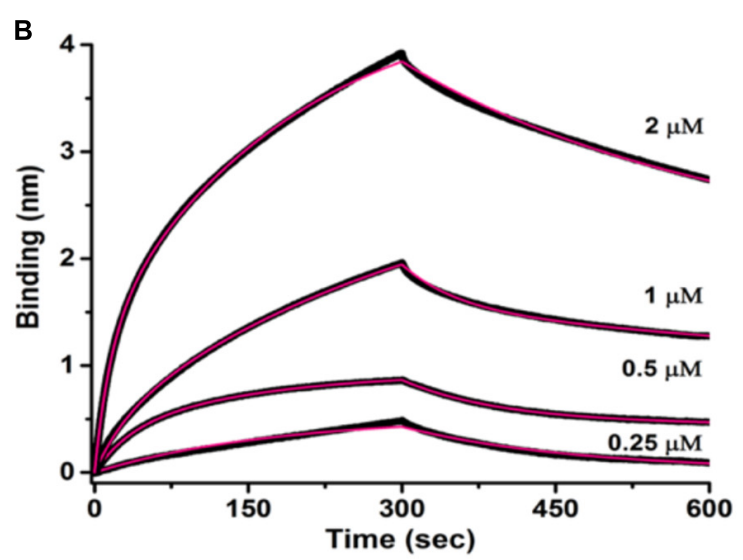

D

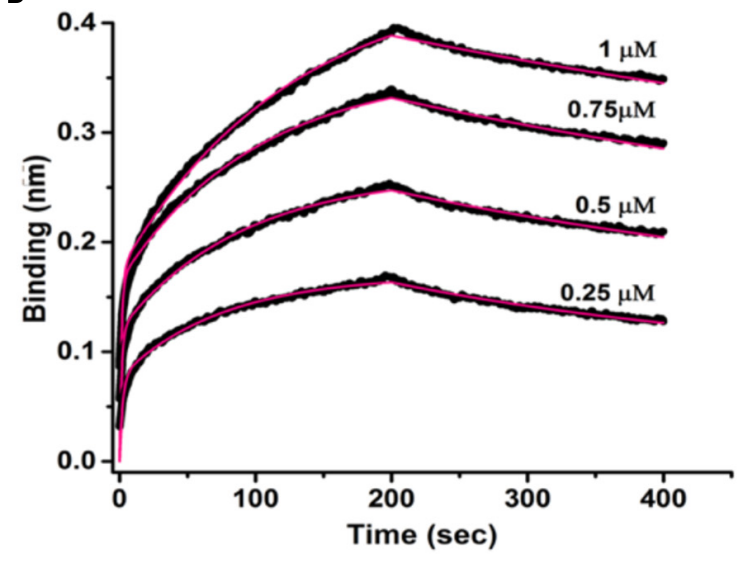

FIGURE 5 | DNA binding property of YeeF-CT ${ }^{(\mathrm{M})}$ and YeeF-CT ${ }^{(\mathrm{M})}$ /YezG. (A) YeeF-CT ${ }^{(\mathrm{M})}$ exhibits non-specific DNA binding property. A smear of retarded DNA can be visualized in the agarose gel. With an increase in the concentration of YeeF-CT ${ }^{(M)}$ the mobility of the DNA-protein complex is retarded and is stuck in the well. (B) BLI kinetics showing binding of non-specific dsDNA with YeeF-CT ${ }^{(M)}$. (C,D) Binding of YezG did not alter the DNA binding property of YeeF-CT(M) . (C) The agarose gel electrophoresis of DNA incubated with different concentrations of YeeF-CT ${ }^{(M)} /$ YezG show retardation in DNA mobility. (D) BLI kinetics showing binding of non-specific dsDNA with YeeF-CT ${ }^{(\mathrm{M})}$ YezG. Both raw sensorgrams (black lines) and fitted curves (red lines) are shown in the figure.

from E. coli O157:H7 (Morse et al., 2012) and colicin (E3, 7, $8,9)$ where they utilize the exosite and neutralize the toxins indirectly by distorting the active site and preventing binding of the substrate (Ko et al., 1999; Mosbahi et al., 2004; Lancaster et al., 2008; Meenan et al., 2010). However, there are no reports where the toxin/immunity complex also interacts with the DNA substrate. The spatial exclusion of immunity protein and substrate binding sites probably provides new opportunities to evolve novel protein-protein interaction interfaces while maintaining the integrity of the active site and thus toxic activity. This provides a selective advantage over the neighboring bacteria having closely related toxins.

Using AUC experiments, we are able to unambiguously assign oligomeric states of YeeF-CT and YezG and binding stoichiometry of the complex. To the best of our knowledge, this is the first polymorphic toxin which exists as a homodimer in solution. It is still unclear whether dimerization is required for active site formation at the interface, or enhances stability, or is involved in some other function. Future structural studies may help us in understanding this better. The oligomeric state of YeeF-CT and YeeF-CT/YezG is unique among structurally characterized toxin/immunity pairs from other PTS (Morse et al., 2012, 2015; Johnson et al., 2016a,b). To further investigate the thermodynamic parameters of this interaction, we used ITC. Nanomolar range affinity of YeeF-CT/YezG is comparable to other PTS, CdiA-CT $536 / \mathrm{CdiI}^{536}\left(\mathrm{~K}_{\mathrm{D}}=26.4 \pm 2.1 \mathrm{nM}\right)$ (Kaundal et al., 2016), CdiA-CT ${ }_{I I}{ }^{B p 1026 b} / C d_{I I}{ }^{B p 1026 b}\left(K_{D}=21.1 \pm 9 \mathrm{nM}\right)$ and CdiA-CTO11 ${ }^{\mathrm{EC} 869} / \mathrm{CdiI}^{\mathrm{EC} 869}\left(\mathrm{~K}_{\mathrm{D}}=17.8 \pm 7 \mathrm{nM}\right.$ ) (Morse et al., 2012) studies on Gram-negative bacteria. The ITC data suggests an allosteric mode of binding as two sites on YeeF-CT dimer have $\sim 3.5$-fold difference in affinity for YezG molecules. This suggests that the binding of one YezG molecule may probably lead to consequent conformational rearrangements, allosterically reducing the affinity for the second molecule. To summarize, we have discovered a metal ion(s) dependent bacterial DNase. Our data also suggests that YezG is an exosite inhibitor of YeeF-CT, where the preformed toxin/immunity complex retains the ability to bind to substrate DNA (Figure 5). Our lab is currently working on solving the high-resolution structures of YeeF-CT/YezG, 
YeeF-CT/YezG/DNA and YeeF-CT/DNA complexes to better understand the molecular basis for DNA binding and unravel the allosteric mechanism involved in toxin/immunity protein interactions.

\section{DATA AVAILABILITY STATEMENT}

The datasets generated for this study are available on request to the corresponding author.

\section{AUTHOR CONTRIBUTIONS}

KT conceived the idea. KT, SK, and AD planned the experiments, analyzed the data and wrote the manuscript. SK, AD, and GK performed the experiments.

\section{FUNDING}

The work was supported by the Council of Scientific \& Industrial Research and Department of Science and Technology,

\section{REFERENCES}

Anjem, A., Varghese, S., and Imlay, J. A. (2009). Manganese import is a key element of the OxyR response to hydrogen peroxide in Escherichia coli. Mol. Microbiol. 72, 844-858. doi: 10.1111/j.1365-2958.2009.06699.x

Aoki, S. K., Diner, E. J., de Roodenbeke, C. T., Burgess, B. R., Poole, S. J., Braaten, B. A., et al. (2010). A widespread family of polymorphic contactdependent toxin delivery systems in bacteria. Nature 468, 439-442. doi: 10. 1038/nature09490

Aoki, S. K., Pamma, R., Hernday, A. D., Bickham, J. E., Braaten, B. A., and Low, D. A. (2005). Contact-dependent inhibition of growth in Escherichia coli. Science 309, 1245-1248. doi: 10.1126/science.1115109

Batot, G., Michalska, K., Ekberg, G., Irimpan, E. M., Joachimiak, G., Jedrzejczak, R., et al. (2017). The CDI toxin of Yersinia kristensenii is a novel bacterial member of the RNase A superfamily. Nucleic Acids Res. 45, 5013-5025. doi: $10.1093 / \mathrm{nar} / \mathrm{gkx} 230$

Cayley, S., Lewis, B. A., Guttman, H. J., and Record, M. T. Jr. (1991). Characterization of the cytoplasm of Escherichia coli $\mathrm{K}-12$ as a function of external osmolarity. Implications for protein-DNA interactions in vivo. J. Mol. Biol. 222, 281-300. doi: 10.1016/0022-2836(91)90212-o

Diner, E. J., Beck, C. M., Webb, J. S., Low, D. A., and Hayes, C. S. (2012). Identification of a target cell permissive factor required for contact-dependent growth inhibition (CDI). Genes Dev. 26, 515-525. doi: 10.1101/gad.182345.111

Hayes, C. S., Koskiniemi, S., Ruhe, Z. C., Poole, S. J., and Low, D. A. (2014). Mechanisms and biological roles of contact-dependent growth inhibition systems. Cold Spring Harb. Perspect. Med. 4:a010025. doi: 10.1101/cshperspect. a010025

Hibbing, M. E., Fuqua, C., Parsek, M. R., and Peterson, S. B. (2010). Bacterial competition: surviving and thriving in the microbial jungle. Nat. Rev. Microbiol. 8, 15-25. doi: 10.1038/nrmicro2259

Ho, T. Y., Wu, S. L., Hsiang, C. H., Chang, T. J., and Hsiang, C. Y. (2000). Identification of a DNA-binding domain and an active-site residue of pseudorabies virus DNase. Biochem. J. 346(Pt 2), 441-445. doi: 10.1042/ bj3460441

Holberger, L. E., Garza-Sanchez, F., Lamoureux, J., Low, D. A., and Hayes, C. S. (2012). A novel family of toxin/antitoxin proteins in Bacillus species. FEBS Lett. 586, 132-136. doi: 10.1016/j.febslet.2011.12.020

Jamet, A., Charbit, A., and Nassif, X. (2018). Antibacterial toxins: gram-positive bacteria strike back! Trends Microbiol. 26, 89-91. doi: 10.1016/j.tim.2017. 11.003
India grants to KT. KT was a recipient of Innovative Young Biotechnologist Award 2011, Department of Biotechnology, India. SK was a recipient of UGC senior research fellowship. $\mathrm{AD}$ was a recipient of DBT senior research fellowship. GK was a DST-INSPIRE fellow.

\section{ACKNOWLEDGMENTS}

KT and SK would like to thank Deepak Bhatt, Vineet Kumar, and Prashant Singh, CSIR-IMTECH for their valuable help in confocal microscopy and BLI experiments. We also thank Mr. Himanshu Malhotra and Mr. Randeep for their help in TEM experiments. The authors thank Mr. Surinder Singh for his technical support and assistance.

\section{SUPPLEMENTARY MATERIAL}

The Supplementary Material for this article can be found online at: https://www.frontiersin.org/articles/10.3389/fmicb. 2020.00095/full\#supplementary-material

Jamet, A., Jousset, A. B., Euphrasie, D., Mukorako, P., Boucharlat, A., Ducousso, A., et al. (2015). A new family of secreted toxins in pathogenic Neisseria species. PLoS Pathog. 11:e1004592. doi: 10.1371/journal.ppat.1004592

Jamet, A., and Nassif, X. (2015). New players in the toxin field: polymorphic toxin systems in bacteria. mBio 6:e00285-15. doi: 10.1128/mBio.00285-215

Jamet, A., Touchon, M., Ribeiro-Goncalves, B., Carrico, J. A., Charbit, A., Nassif, X., et al. (2017). A widespread family of polymorphic toxins encoded by temperate phages. BMC Biol. 15:75. doi: 10.1186/s12915-017-0415-411

Johnson, P. M., Beck, C. M., Morse, R. P., Garza-Sanchez, F., Low, D. A., Hayes, C. S., et al. (2016a). Unraveling the essential role of CysK in CDI toxin activation. Proc. Natl. Acad. Sci. U.S.A. 113, 9792-9797. doi: 10.1073/pnas. 1607112113

Johnson, P. M., Gucinski, G. C., Garza-Sanchez, F., Wong, T., Hung, L. W., Hayes, C. S., et al. (2016b). Functional diversity of cytotoxic tRNase/immunity protein complexes from Burkholderia pseudomallei. J. Biol. Chem. 291, 19387-19400. doi: 10.1074/jbc.M116.736074

Kaundal, S., Uttam, M., and Thakur, K. G. (2016). Dual role of a biosynthetic enzyme, CysK, in contact dependent growth inhibition in bacteria. PLoS One 11:e0159844. doi: 10.1371/journal.pone.0159844

Kaur, G., Sengupta, S., Kumar, V., Kumari, A., Ghosh, A., Parrack, P., et al. (2014). Novel MntR-independent mechanism of manganese homeostasis in Escherichia coli by the ribosome-associated protein HflX. J. Bacteriol. 196, 2587-2597. doi: 10.1128/JB.01717-1714

Keller, L., and Surette, M. G. (2006). Communication in bacteria: an ecological and evolutionary perspective. Nat. Rev. Microbiol. 4, 249-258. doi: 10.1038/ nrmicro1383

Kleanthous, C., and Walker, D. (2001). Immunity proteins: enzyme inhibitors that avoid the active site. Trends Biochem. Sci. 26, 624-631. doi: 10.1016/s09680004(01)01941-7

Ko, T. P., Liao, C. C., Ku, W. Y., Chak, K. F., and Yuan, H. S. (1999). The crystal structure of the DNase domain of colicin E7 in complex with its inhibitor Im7 protein. Structure 7, 91-102. doi: 10.1016/s0969-2126(99)80012-4

Koskiniemi, S., Lamoureux, J. G., Nikolakakis, K. C., t’Kint de Roodenbeke, C., Kaplan, M. D., Low, D. A., et al. (2013). Rhs proteins from diverse bacteria mediate intercellular competition. Proc. Natl. Acad. Sci. U.S.A. 110, 7032-7037. doi: $10.1073 /$ pnas. 1300627110

Lancaster, L. E., Savelsbergh, A., Kleanthous, C., Wintermeyer, W., and Rodnina, M. V. (2008). Colicin E3 cleavage of 16S rRNA impairs decoding and accelerates tRNA translocation on Escherichia coli ribosomes. Mol. Microbiol. 69, 390-401. doi: 10.1111/j.1365-2958.2008.06283.x 
Laue, T. M., Shah, B. D., Ridgeway, T. M., and Pelletier, S. L. (1992). "Computeraided interpretation of analytical sedimentation data for proteins," in Analytical Ultracentrifugation in Biochemistry and Polymer Science, eds S. E. Harding, A. J. Rowe, and J. C. Horton, (Cambridge: Royal Society of Chemistry), 90-25.

Meenan, N. A., Sharma, A., Fleishman, S. J., Macdonald, C. J., Morel, B., Boetzel, R., et al. (2010). The structural and energetic basis for high selectivity in a high-affinity protein-protein interaction. Proc. Natl. Acad. Sci. U.S.A. 107, 10080-10085. doi: 10.1073/pnas.0910756107

Morse, R. P., Nikolakakis, K. C., Willett, J. L., Gerrick, E., Low, D. A., Hayes, C. S., et al. (2012). Structural basis of toxicity and immunity in contactdependent growth inhibition (CDI) systems. Proc. Natl. Acad. Sci. U.S.A. 109, 21480-21485. doi: 10.1073/pnas.1216238110

Morse, R. P., Willett, J. L., Johnson, P. M., Zheng, J., Credali, A., Iniguez, A., et al. (2015). Diversification of beta-augmentation interactions between CDI Toxin/immunity proteins. J. Mol. Biol. 427, 3766-3784. doi: 10.1016/j.jmb.2015. 09.020

Mosbahi, K., Walker, D., Lea, E., Moore, G. R., James, R., and Kleanthous, C. (2004). Destabilization of the colicin E9 Endonuclease domain by interaction with negatively charged phospholipids: implications for colicin translocation into bacteria. J. Biol. Chem. 279, 22145-22151. doi: 10.1074/jbc.M400402200

Ogier, J. C., Duvic, B., Lanois, A., Givaudan, A., and Gaudriault, S. (2016). A New Member of the Growing Family of Contact-Dependent Growth Inhibition Systems in Xenorhabdus doucetiae. PLoS One 11:e0167443. doi: 10.1371/ journal.pone.0167443

Poole, S. J., Diner, E. J., Aoki, S. K., Braaten, B. A., t'Kint de Roodenbeke, C., Low, D. A., et al. (2011). Identification of functional toxin/immunity genes linked to contact-dependent growth inhibition (CDI) and rearrangement hotspot (Rhs) systems. PLoS Genet. 7:e1002217. doi: 10.1371/journal.pgen.1002217

Schuck, P., Perugini, M. A., Gonzales, N. R., Howlett, G. J., and Schubert, D. (2002). Size-distribution analysis of proteins by analytical ultracentrifugation: strategies and application to model systems. Biophys. J. 82, 1096-1111. doi: 10.1016/S0006-3495(02)75469-75466

Visick, K. L., and Fuqua, C. (2005). Decoding microbial chatter: cell-cell communication in bacteria. J. Bacteriol. 187, 5507-5519. doi: 10.1128/JB.187. 16.5507-5519.2005

Waters, C. M., and Bassler, B. L. (2005). Quorum sensing: cell-to-cell communication in bacteria. Annu. Rev. Cell. Dev. Biol. 21, 319-346. doi: 10. 1146/annurev.cellbio.21.012704.131001

Whitney, J. C., Peterson, S. B., Kim, J., Pazos, M., Verster, A. J., Radey, M. C., et al. (2017). A broadly distributed toxin family mediates contact-dependent antagonism between gram-positive bacteria. eLife 6:e26938. doi: 10.7554/eLife. 26938

Zhang, D., de Souza, R. F., Anantharaman, V., Iyer, L. M., and Aravind, L. (2012). Polymorphic toxin systems: comprehensive characterization of trafficking modes, processing, mechanisms of action, immunity and ecology using comparative genomics. Biol. Direct. 7:18. doi: 10.1186/1745-6150-7-18

Zhang, D., Iyer, L. M., and Aravind, L. (2011). A novel immunity system for bacterial nucleic acid degrading toxins and its recruitment in various eukaryotic and DNA viral systems. Nucleic Acids Res. 39, 4532-4552. doi: 10.1093/nar/ gkr036

Conflict of Interest: The authors declare that the research was conducted in the absence of any commercial or financial relationships that could be construed as a potential conflict of interest.

Copyright (C) 2020 Kaundal, Deep, Kaur and Thakur. This is an open-access article distributed under the terms of the Creative Commons Attribution License (CC BY). The use, distribution or reproduction in other forums is permitted, provided the original author(s) and the copyright owner(s) are credited and that the original publication in this journal is cited, in accordance with accepted academic practice. No use, distribution or reproduction is permitted which does not comply with these terms. 